Federal Reserve Bank of Dallas

Globalization and Monetary Policy Institute

Working Paper No. 274

http://www.dallasfed.org/assets/documents/institute/wpapers/2016/0274.pdf

\title{
The Market Resources Method for Solving Dynamic Optimization Problems*
}

\author{
Ayşe Kabukçuoğlu \\ Koç University \\ Enrique Martínez-García \\ Federal Reserve Bank of Dallas
}

June 2016

\begin{abstract}
We introduce the market resources method (MRM) for solving dynamic optimization problems. MRM extends Carroll's (2006) endogenous grid point method (EGM) for problems with more than one control variable using policy function iteration. The MRM algorithm is simple to implement and provides advantages in terms of speed and accuracy over Howard's policy improvement algorithm. Codes are available.
\end{abstract}

JEL codes: C6, C61, C63, C68

\footnotetext{
* Ayşe Kabukçuoğlu, Koç University, Rumelifeneri Yolu, Sariyer, Istanbul 34450 Turkey. akabukcuoglu@ku.edu.tr. Enrique Martínez-García, Federal Reserve Bank of Dallas, Research Department, 2200 N. Pearl Street, Dallas, TX 75201. 214-922-5194. enrique.martinez-garcia@dal.frb.org. We would like to thank Zeynep Kabukçuoğlu for providing invaluable suggestions, and Andy Glover for helpful comments. All errors are ours alone. The views in this paper are those of the authors and do not necessarily reflect the views of the Federal Reserve Bank of Dallas or the Federal Reserve System.
} 


\section{Introduction}

Most dynamic models in macroeconomics are in the class of nonlinear rational expectations models which are complex and rich in structure, and do not exhibit a closed-form, analytical solution. A solution, if it exists, can be obtained only through numerical techniques. The literature has gained momentum in recent years in producing new methods that attain improved levels of accuracy, speed and/or practicality to implement (See Maliar and Maliar (2014) for an extensive review on recent techniques for the solution of high-dimensional problems). One such technique by Carroll (2006), the Endogenous Gridpoints Method (EGM), offers an insight to reduce the cost of conventional value function iteration (VFI).

The main idea behind EGM is to consider the future endogenous state variable fixed, and the current endogenous state variable unknown, unlike the standard approach that takes the current state variable as given, and solve forward to find the optimal state variable tomorrow. In an application to the standard stochastic neoclassical growth model, a solution under EGM involves finding decision rules as a function of next period's capital as opposed to the conventional VFI that defines decision rules over current period's capital. This generates a faster solution than the standard approach as the consumption Euler equation is usually nonlinear in next period's capital but linear in current period's capital.

The literature has developed ways of solving relatively more complex dynamic programming problems in this spirit Carroll (2006). ${ }^{1}$ Among those, for instance, Barillas and Fernandez-Villaverde (2007) extend the solution method to problems with labor-leisure choice, Hintermaier and Koeniger (2010) and Fella (2014) suggest solution methods for a model with occasionally binding constraints and a decision on durables and non-durables, White (2015) considers the theoretical characterization of EGM with a multi-dimensional setting and under value function iteration. ${ }^{2}$

In this paper, we take a different route and develop a method that relies on policy function iteration, namely the Market Resources Method (MRM), paying attention primarily to the solution of problems with labor-leisure choice directly from the first order conditions. Working on a collection of equations rather than iterating on the value function might help avoid some well-known computational problems arising from approximating the value function and the curse of dimensionality. The MRM algorithm involves an approximation method, too-albeit an interpolation of the policy function only. Our technique, which relies mostly on algebraic solutions, enables the use of a coarse grid.

Our method particularly blends Coleman (1990)'s time iteration with Carroll (2006)'s EGM to exploit the speed and accuracy gains in these methods in major ways. Coleman (1990) suggests iterating on policy functions directly using the Euler equation, but the grids are defined in a conventional way, and therefore, in each iteration, a nonlinear equation solver is needed to find current consumption (and there is no laborleisure choice). First, changing the time convention as in Carroll (2006)'s EGM to define the grid points over next period's endogeneous state variable rather than the current helps avoid the excessive burden of nonlinear equation solvers. Second, we can consider a change of variables in the resource constraint to define a new state variable, 'market resources', i.e. the sum of output and capital after depreciation, and this helps us solve for current-period capital and labor only once and after convergence is achieved rather

\footnotetext{
${ }^{1}$ In the original version of EGM, Carroll (2006) considers a model with one continuous control variable and one-dimensional state space.

${ }^{2}$ Another approach to reduce the cost of dynamic programming methods is coined by Maliar and Maliar (2013) with the envelope condition method (ECM), which simplifies the rootfinding procedure and produces highly accurate and fast results that are comparable to the EGM in the solution of the stochastic growth model with labor-leisure choice.
} 
than in every iteration, which reduces computation time dramatically by avoiding, again, these extra rootfinding procedures. This change of variables in the spirit of Carroll (2006) would not be possible in a VFIbased technique that has endogenous labor since an additional decision on labor-leisure requires a guess of a policy function. This will be explained in detail in the subsequent sections of the paper. Hence, this short-cut appears to be feasible only in a policy function iteration-based method.

The literature suggests that in theory, it is possible to obtain equivalent solutions under standard value function iteration and time iteration (see Coleman (1990) in the stochastic growth model application and in particular, Rendahl (2015) for a generalization with occasionally binding constraints), hence one method is not more advantagous than another. But key differences arise in the implementation of these methods. Despite their speed, a caveat in Euler equation-based techniques is convergence issues unless one starts with an educated initial guess, which might be a problem, for instance, with the parameterized expectations algorithm of den Haan and Marcet (1990). But with the proposed method we show convergence is achieved even in the absence of an educated guess, although it requires more computing time. We then compare the speed and accuracy gains from MRM to those of the Howard's policy improvement algorithm (Howard (1960)). Our proposed method yields dramatic speed and accuracy gains over the standard policy function iteration (PFI).

The PFI is a high threshold to pass in terms of speed and accuracy since it is known to be a powerful method that makes use of each new computed policy function by computing the value of using that policy forever, usually requiring a smaller number of steps for convergence compared to the standard VFI (Santos and Rust (2003)). On the other hand, the standard VFI assumes that the policy function is used only for once and hence becomes slower compared to PFI.

Our experiment with MRM is based on the stochastic neoclassical growth model with labor-leisure choice. An additional static choice variable might complicate the solution technique significantly, and MRM provides a faster and/or more accurate technique compared to PFI at this level of complexity. Similar levels of accuracy, as measured by first order condition errors, can be attained with 50 grid points in MRM and 1000 grid points in PFI, finding a solution to the problem in 2.2 CPU seconds and 642 CPU seconds, respectively.

\section{The Stochastic Neoclassical Growth Model}

In order to assess the performance of the market resources method, we consider the stochastic neoclassical growth model as described in Cooley and Prescott (1995). Starting with Kydland and Prescott (1982), this has been one of the most-widely studied models in macroeconomics from which many of the existing general equilibrium models have evolved. While we show that it is possible to exploit the efficiency and accuracy gains of MRM in a model with no kinks or constraints, the method could be applied to solve workhorse macro models in the heterogeneous-agent incomplete markets literature in the spirit of Bewley (1986), Huggett (1993), Aiyagari (1994), and Imrohoroglu (1989). ${ }^{3}$

\footnotetext{
${ }^{3}$ Indeed, there are earlier applications of EGM based on policy function iteration in non-Pareto optimal economies such as Guerrieri and Lorenzoni (2015) and Kabukcuoglu (2015). The former abstracts away from physical capital and the latter from elastic labor. The MRM approach provides an advantage in problems that handles a decision for both capital and labor.
} 


\subsection{The Model}

The expected life-time uility of a representative household is given by

$$
U=\mathbb{E}_{0} \sum_{t=0}^{\infty} \beta^{t} u\left(c_{t}, 1-l_{t}\right)
$$

where $\beta \in(0,1)$ is the discount factor, $c_{t}$ is consumption, $l_{t} \in[0,1]$ is labor supply (and hence, $1-l_{t}$ is defined as leisure). The period utility function $u(\cdot)$ is strictly increasing, strictly concave and continuously differentiable in the two arguments. The single aggregate output in the economy, $y_{t}$ is produced using aggregate capital, $k_{t}$, and aggregate labor, $l_{t}$, according to a constant returns to scale production function, $y_{t}=e^{z_{t}} F\left(k_{t}, l_{t}\right)$. The total factor productivity (TFP), $z_{t}$, is governed by an $n$-state $(n<\infty)$ first-order Markov process defined with an $n \times n$ transition probability matrix $\Pi=\left[\pi_{i j}\right]$, where $\pi_{i j}=\operatorname{Pr}\left(z_{t+1}=z_{j} \mid z_{t}=z_{i}\right)$. All elements of $\Pi$ are non-negative and each row sums up to 1 . In any given period, the decisions are made after observing the shock $z_{t}$.

Capital depreciates at rate $\delta \in[0,1]$ in each period. Hence the resource constraint for the social planner's problem can be written as

$$
c_{t}+k_{t+1}=e^{z_{t}} F\left(k_{t}, l_{t}\right)+(1-\delta) k_{t} .
$$

In this model, one can appeal to the first and second welfare theorems to solve for the social planner's problem - the allocation is Pareto-efficient and can be decentralized in a standard way through a competitive markets' equilibrium. Therefore, the sequential dynamic optimization problem is to maximize (1) subject to the resource constraint in (2), the law of motion for TFP shocks and the given initial conditions for the stoch of capital, $k_{0}$, and TFP, $z_{0}$.

\subsection{Equilibrium Characterization}

The equilibrim for the social planner's problem can be characterized by an Euler equation, an intratemporal consumption-leisure condition, and the resource constraint, i.e.

$$
\begin{aligned}
& u_{c}\left(c_{t}, 1-l_{t}\right)=\beta \mathbb{E}_{z_{t+1} \mid z_{t}} u_{c}\left(c_{t+1}, 1-l_{t+1}\right)\left[e^{z_{t+1}} F_{k}\left(k_{t+1}, l_{t+1}\right)+1-\delta\right] \\
& u_{c}\left(c_{t}, 1-l_{t}\right) e^{z_{t}} F_{l}\left(k_{t}, l_{t}\right)=u_{l}\left(c_{t}, 1-l_{t}\right) \\
& c_{t}+k_{t+1}=e^{z_{t}} F\left(k_{t}, l_{t}\right)+(1-\delta) k_{t}
\end{aligned}
$$

plus the law of motion for the technology shocks, the initial conditions on the stock of capital and the technology shock, and the corresponding transversality conditions.

\subsection{The Market Resources Method (MRM)}

In this section, we show how to solve the stochastic neoclassical growth model with labor-leisure choice based on MRM. This approach requires that we use the conditions (3), (4), and (5) as well as the law of motion for $z_{t}$ to obtain the solutions for consumption, $c_{t}^{*}$, labor, $l_{t}^{*}$, and capital, $k_{t}^{*}$, given capital tomorrow, $k_{t+1}$, and today's productivity shock, $z_{t}$ and is based on policy function iteration. This means that given tomorrow's capital $k_{t+1}$, we pin down today's capital, $k_{t}^{*}$, and these values are often off the grid as the method involves algebraic solutions. Setting the time convention for the grid points this way to recover the 
current endogenous state variable is in line with the endogeneous gridpoint method (EGM) introduced by Carroll (2006). In what follows, we explain how our MRM differs from and builds on Carroll's EGM.

Carroll (2006)'s EGM offers a solution for a problem with no static choice variables using value function iteration. ${ }^{4}$ To this end, he considers a helpful switch of variables by defining a new variable called 'market resources' (or 'cash-on-hand' for a micro interpretation), $m_{t}$ where

$$
m_{t}=c_{t}+k_{t+1}=e^{z_{t}} F\left(k_{t}, 1\right)+(1-\delta) k_{t} .
$$

Then he considers a Bellman equation of the form:

$$
V\left(m_{t}, z_{t}\right)=\max _{k_{t+1}}\left\{u\left(c_{t}\right)+\beta \mathbb{E}_{z_{t+1} \mid z_{t}} V\left(m_{t+1}, z_{t+1}\right)\right\}
$$

and the FOC for the problem looks as follows:

$$
u^{\prime}\left(c_{t}\right)=\beta \mathbb{E}_{z_{t+1} \mid z_{t}}\left[V_{m}\left(m_{t+1}, z_{t+1}\right)\right] .
$$

Notice that with inelastic labor supply, it is possible to fix a grid of $m_{t+1}$ using the grids of $k_{t+1}$ as in Carroll (2006). This helps construct the right hand side of equation (8) on a fixed grid. Then it is easy to pin down $c_{t}$ algebraically given an explicit specification for $u^{\prime}\left(c_{t}\right)$ and compute $m_{t}=c_{t}+k_{t+1}$. This completes the final step to iterate on the value function without a need to compute $k_{t}$ on every iteration using a nonlinear equation solver. If the labor supply were elastic however, it would not be possible to fix the grid $m_{t}=e^{z_{t}} F\left(k_{t}, l_{t}\right)+(1-\delta) k_{t}$ as it would require us to know the decision rule for labor. ${ }^{5}$

The key idea of the MRM, as the name suggests, is to take advantage of a switch of variables even when labor supply is elastic using iteration on Euler equation. Again, we define market resources similarly for the current period, $m_{t}=c_{t}+k_{t+1}=e^{z_{t}} F\left(k_{t}, l_{t}\right)+(1-\delta) k_{t}$ and for the next period $m_{t+1}=e^{z_{t+1}} F\left(k_{t+1}, l_{t+1}\right)+$ $(1-\delta) k_{t+1}$. This redefinition enables us to sidestep the burden of using a nonlinear equation solver to find this period's capital and labor in every iteration. Therefore, we can solve for the decision rules for $k_{t}$ and $l_{t}$ only once, in the final step, which is not feasible under VFI-based techniques since we cannot use the transformation of market resources.

Once we consider this transformation, the time-invariant decision rules we aim to solve for are $c_{t}=$ $\tilde{g}_{c}\left(m_{t}^{*}, z_{t}\right)=g_{c}\left(k_{t}^{*}, z_{t}\right), l_{t}=\tilde{g}_{l}\left(m_{t}^{*}, z_{t}\right)=g_{l}\left(k_{t}^{*}, z_{t}\right)$ and $k_{t+1}=\tilde{g}_{k}\left(m_{t}^{*}, z_{t}\right)=g_{k}\left(k_{t}^{*}, z_{t}\right)$.

In MRM, our first goal is to recover $c_{t}$ from (3), (4), and (5). When the utility function is separable in consumption and leisure, this is possible once we know $k_{t+2}, l_{t+1}$ and $c_{t+1}$ given $\left(k_{t+1}, z_{t+1}\right)$. Therefore, we start with an initial guess for $k_{t+2}=\tilde{g}_{k}\left(m_{t+1}, z_{t+1}\right)=g_{k}\left(k_{t+1}, z_{t+1}\right)$. Notice that $m_{t+1}$ is defined over the grid points $k_{t+1}$, hence we can base our guess directly on these grid points. Since the guess is updated based on an interpolation method, the resulting $k_{t+2}^{*}$ values will lie off the grid, just like $k_{t}^{*}$.

Next, with the guess for $k_{t+2}$ and for a pair $\left(k_{t+1}, z_{t+1}\right)$ we can solve for $l_{t+1}$ from a nonlinear equation. In particular, we need to solve the following equation that combines the intratemporal FOC (4) and the

\footnotetext{
${ }^{4} \mathrm{He}$ also considers a finite-horizon economy while we focus on an infinite-horizon problem.

${ }^{5}$ Barillas and Fernandez-Villaverde (2007) deal with this problem with labor-leisure choice by proposing an algorithm in two steps. First, they fix the labor supply at the steady state level and apply the standard EGM algorithm to obtain a value function. This value function serves as a very accurate guess in a standard value function iteration which is employed in the second step to obtain decision rules for savings, consumption and labor.
} 
resource constraint (5):

$$
g_{k}\left(k_{t+1}, z_{t+1}\right)-e^{z_{t+1}} F\left(k_{t+1}, l_{t+1}\right)-(1-\delta) k_{t+1}+u_{c}^{-1}\left[u_{l}\left(1-l_{t+1}\right) / e^{z_{t+1}} F_{l}\left(k_{t+1}, l_{t+1}\right)\right]=0 .
$$

Notice that since $k_{t+2}=\tilde{g}_{k}\left(k_{t+1}, z_{t+1}\right)$, we can consider a solution for next period's labor at any $\left(k_{t+1}, z_{t+1}\right)$ pair, i.e. $l_{t+1}=g_{l}\left(k_{t+1}, z_{t+1}\right)$ from (9). Then, from the resource constraint (5), we can pin down next period's consumption,

$$
c_{t+1}=-\tilde{g}_{k}\left(k_{t+1}, z_{t+1}\right)+e^{z_{t+1}} F\left(k_{t+1}, g_{l}\left(k_{t+1}, z_{t+1}\right)\right)+(1-\delta) k_{t+1} .
$$

Since the utility function is separable in consumption and leisure, and using $c_{t+1}, l_{t+1}$, as well as the $k_{t+1}$ values on the grid, we can solve for $c_{t}$ directly from the Euler equation,

$$
c_{t}=u_{c}^{-1}\left(\beta \mathbb{E}_{z_{t+1} \mid z_{t}} u_{c}\left(g_{c}\left(k_{t+1}, z_{t+1}\right)\right)\left[e^{z_{t+1}} F_{k}\left(k_{t+1}, g_{l}\left(k_{t+1}, z_{t+1}\right)\right)+1-\delta\right]\right) .
$$

The implied market resources $m_{t}$, for this period using the solution for $c_{t}$ in (11), such that

$$
m_{t}=c_{t}+k_{t+1} .
$$

Using implied labor from (9) enables us to find also the market resources for the next period,

$$
m_{t+1}=e^{z_{t+1}} F\left(k_{t+1}, g_{l}\left(k_{t+1}, z_{t+1}\right)\right)+(1-\delta) k_{t+1} .
$$

Now we will update our guess for $k_{t+2}$ without finding $k_{t}$ or $l_{t}$ and only using market resources. The main logic of MRM is based on the idea that by using (12), we can consider $k_{t+1}$ as a time-invariant function of $m_{t}$ and $z_{t}$, i.e. $k_{t+1}=\tilde{g}_{k}\left(m_{t}, z_{t}\right)$. This implies that $k_{t+2}$ can be expressed as a function of next period's states, i.e. $k_{t+2}=\tilde{g}_{k}\left(m_{t+1}, z_{t+1}\right)$. We can then use an interpolation method to interpolate $k_{t+2}$ on $m_{t+1}$ using $m_{t}$ and $z_{t}$. With the resulting values for $k_{t+2}$ we update our guess until a stopping criterion is satisfied. And once convergence is achieved with a solution to $c_{t}^{*}$, we can find $l_{t}^{*}$ and $k_{t}^{*}$ jointly from (4) and (5) using a nonlinear equation solver.

The iteration on the Euler equation based on a policy function guess in the MRM algorithm is closely related to Coleman (1990)'s time iteration method whose aim is to solve a fixed-point equation in the form $c=F(c)$ where $c$ is the optimal consumption function and $F$ is derived from the intertemporal Euler equation. In that regard, the uniqueness of the solution and the convergence properties of our algorithm are similar to that in Coleman (1990), where time-iteration is shown to be equivalent to value function iteration. Rendahl (2015) provides an extensive theoretical discussion on the equivalence of a solution in multi-dimensional problems with inequality constraints, with a comparison of VFI, PFI and time iteration. ${ }^{6}$

The MRM algorithm: We define the grid points for tomorrow's capital, $\mathcal{G}_{k_{t+1}} \equiv\left\{k_{1}, k_{2}, \ldots, k_{M}\right\}$ and use the Tauchen (1986) approximation method to obtain the discretized stochastic process for the total factor productivity shocks, defined with grid points $\mathcal{G}_{z_{t}} \equiv\left\{z_{1}, z_{2}, \ldots, z_{N}\right\}$ with the associated transition probability matrix $\Pi$. The steps of the algorithm are described as follows:

1. Set $i=0$ and make a policy function guess $k_{t+2}=\tilde{g}_{k}^{i}\left(m_{t+1}, z_{t+1}\right)=g_{k}^{i}\left(k_{t+1}, z_{t+1}\right)$. We start with a

\footnotetext{
${ }^{6} \mathrm{He}$ then numerically evaluates an iterative scheme on the Euler equation, where the derivative of the value function is updated along with the savings decision rule in a problem with no endogenous labor supply decision.
} 
guess that sets $k_{t+2}=\mathrm{k}^{s s}$, the steady-state value of capital for all pairs $\left(k_{t+1}, z_{t+1}\right) \in \mathcal{G}_{k_{t+1}} \times \mathcal{G}_{z_{t}}$. (We can rely on an arbitrary guess as well, but but it will increase computation time.) Initialize current period market resources such that $m_{t}^{i}\left(k_{t+1}, z_{t+1}\right)=0$ for all $\left(k_{t+1}, z_{t+1}\right) \in \mathcal{G}_{k_{t+1}} \times \mathcal{G}_{z_{t}}$.

2. For each point $g_{k}^{0}\left(k_{t+1}, z_{t+1}\right)$ and associated state $k_{t+1} \in \mathcal{G}_{k_{t+1}}$, solve the nonlinear equation (9) for $l_{t+1}=g_{l}\left(k_{t+1}, z_{t+1}\right)$ using Newton's method, which is quite fast. ${ }^{7}$ Then find $c_{t+1}=g_{c}\left(k_{t+1}, z_{t+1}\right)$ using (10), and $c_{t}$ algebraically from (11) over the grid points $\left(k_{t+1}, z_{t+1}\right)$. Using these decision rules, compute the grid points for market resources $m_{t}^{i+1}$ and $m_{t+1}^{i+1}$ from (12) and (13), respectively.

3. Check if $\sup _{m, n}\left|m_{t}^{i+1}\left(k_{m}, z_{n}\right)-m_{t}^{i}\left(k_{m}, z_{n}\right)\right| \geq 1.0 e^{-6}$. If convergence is not achieved, let $i \rightsquigarrow i+1$ and $m_{t}^{i+1}=m_{t}^{i}$. Update the decision rule for capital using interpolation. In particular, we use piecewise cubic hermite interpolating polynomial ('pchip' in MATLAB) to interpolate $\tilde{g}_{k}^{i+1}\left(m_{t+1}, z_{t+1}\right)$ on $m_{t+1}^{i+1}$ using $m_{t}^{i+1.8}$ Go to step 2.

4. If convergence is achieved, find $l_{t}^{*}$ and $k_{t}^{*}$ jointly from (4) and (5) using a nonlinear equation solver. Here, we use Christopher Sim's csolve function in MATLAB. This step computes $k_{t}^{*}$ given $\left(k_{t+1}, z_{t}\right)$ and these values are off the grid. Hence we know the solutions to the problem, $c_{t}=g_{c}\left(k_{t}^{*}, z_{t}\right)$, $l_{t}=g_{l}\left(k_{t}^{*}, z_{t}\right)$ and $k_{t+1}=g_{k}\left(k_{t}^{*}, z_{t}\right)$.

\subsection{Howard's policy improvement algorithm (PFI)}

The standard policy function iteration (PFI) has been known to be very powerful (see Ljungquist and Sargent (2012), pp. 106-107) as it provides convergence at a quadratic rate, rather than linear, as in the case of value function iteration (Puterman and Brumelle (1979) and Santos and Rust (2003)). It is also a natural benchmark for us since MRM relies on policy function iteration. In this section we will describe how the stochastic neoclassical growth model with labor-leisure choice can be solved based on PFI. In order to iterate on a policy function, we need to express the problem in recursive form, which can be formulated in two ways:

Defining $k_{t+1}$ as our choice variable and treating the intratemporal FOC (4) as an additional constraint and then plugging (9) in for $l_{t} ;(5)$ and (9) for $c_{t}$ in the utility function, we define the dynamic programming problem as follows:

$$
V\left(k_{t}, z_{t}\right)=\max _{k_{t+1}}\left\{u\left(z_{t}, k_{t}, k_{t+1}\right)+\beta \mathbb{E}_{z_{t+1} \mid z_{t}} V\left(k_{t+1}, z_{t+1}\right)\right\} .
$$

Notice that this problem could also be solved by defining the choice variables as $k_{t+1}$ and $l_{t}$ using additional grids for labor. However, the method becomes severely prone to the curse of dimensionality and performs as a relatively worse benchmark to compare against MRM. Furthermore, using PFI with labor grids does not yield a fair comparison with the MRM which only considers a grid for capital.

We use a nonlinear equation solver in order to construct the utility function in three arguments, i.e. $u\left(z_{t}, k_{t}, k_{t+1}\right)$ using grids for $k_{t}$ and $k_{t+1}$ by solving for $l_{t}$ in (9) for any combination of $z_{t}, k_{t}$ and $k_{t+1}$. Then we pick a feasible policy function, $k_{t+1}=g_{k}^{i}\left(k_{t}, z_{t}\right)$ and compute the value associated with forever using

\footnotetext{
${ }^{7}$ Other alternatives for MATLAB such as fsolve and csolve (by Christopher Sims) appear to yield the same results but require more computation time.

${ }^{8}$ Different interpolation techniques are studied in detail in Judd (1998) pp. 216-235.
} 
this policy,

$$
V_{i}\left(g_{k}^{i}\left(k_{t}, z_{t}\right), z_{t+1}\right)=\mathbb{E}_{0} \sum_{t=0}^{\infty} \beta^{t} u\left(k_{t}, g_{k}^{i}\left(k_{t}, z_{t}\right)\right)
$$

with $i=0$. Next, we consider the policy improvement step, where the policy function solves

$$
g_{k}^{i+1}\left(k_{t}, z_{t}\right)=\arg \max _{k_{t+1}}\left\{u\left(z_{t}, k_{t}, k_{t+1}\right)+\beta \mathbb{E}_{z_{t+1} \mid z_{t}} V_{i}\left(g_{k}^{i}\left(k_{t}, z_{t}\right), z_{t+1}\right)\right\}
$$

and iterate over $i$ to convergence on steps (14) and (15).

The PFI algorithm: We define the grid points for capital, $\mathcal{G}_{k} \equiv\left\{k_{1}, k_{2}, \ldots, k_{M}\right\}$, and use the Tauchen (1986) approximation method to obtain the discretized stochastic process for the total factor productivity shocks, defined with grid points $\mathcal{G}_{z_{t}} \equiv\left\{z_{1}, z_{2}, \ldots, z_{N}\right\}$ with the associated transition probability matrix $\Pi$. The steps of the algorithm are described as follows:

1. For each triplet of productivity shock, today's and tomorrow's capital $\left(z_{t}, k_{t}, k_{t+1}\right) \in \mathcal{G}_{k} \times \mathcal{G}_{k} \times \mathcal{G}_{z}$, we construct the matrix $u\left(z_{t}, k_{t}, k_{t+1}\right)$. In order to do so, we solve the nonlinear equation (9) for $l_{t}$ using Newton's method and find $c_{t}$ using $(10)$, over the grid points $\left(k_{t}, z_{t}\right) .^{9}$

2. Set $i=0$ and construct the initial value function $V_{i}\left(k_{t}, z_{t}\right)$. In order for this to be consistent with the initial guess in MRM, we set $V_{i}\left(k_{t}, z_{t}\right)=\frac{u\left(z^{s s}, k^{s s}, k^{s s}\right)}{1-\beta}$ for all $\left(z_{t}, k_{t}, k_{t+1}\right) \in \mathcal{G}_{k} \times \mathcal{G}_{k} \times \mathcal{G}_{z}$.

3. We find the decision rule $\left.g_{k}^{i+1}\left(k_{t}, z_{t}\right)=\arg \max \left\{u\left(z_{t}, k_{t}, k_{t+1}\right)\right\}+\beta \mathbb{E}_{z_{t+1}} \mid z_{t} V_{i}\left(g_{k}^{i}\left(k_{t}, z_{t}\right), z_{t+1}\right)\right\}$.

4. We then need to compute the value of using this policy forever and solve forward the Bellman equation in (15) to find the new value function $V_{i+1}$.

5. Check if $\sup _{m, n}\left|V_{i+1}\left(k_{m}, z_{n}\right)-V_{i}\left(k_{m}, z_{n}\right)\right| \geq 1.0 e^{-6}$. If convergence is not achieved, go to step 2 and let $i \rightsquigarrow i+1$.

6. If convergence is achieved, find $l_{t}=g_{l}\left(k_{t}, z_{t}\right)$ using Newton's method and $c_{t}=g_{c}\left(k_{t}, z_{t}\right)$ from the resource constraint.

Even though the construction of $u\left(z_{t}, k_{t}, k_{t+1}\right)$ in step 1 of PFI is done only once, it requires the use of a numerical solver $N_{k} \times N_{k} \times N_{z}$ times. In MRM however, this procedure is repeated $N_{k} \times N_{z}$ times, but in each iteration when obtaining the labor decision rules in step 2. It requires a quantitative exercise to find out which method is more-time consuming in this step. Our numerical experiments reveal that we need a sufficiently large $N_{k}$ to obtain more accurate results with PFI, and in this case it remains slower than MRM. The rest of the speed advantages in MRM can be attributed to the time-convention in the grid points and the redefinition of the state variable in terms of market resources.

\subsection{Calibration and numerical results}

\subsubsection{Calibration}

Our calibration strategy aims to match the U.S. economy. We mainly follow Barillas and FernandezVillaverde (2007) for the calibration of the parameters of the model except for utility parameterization

\footnotetext{
${ }^{9}$ Christopher Sim's csolve function in MATLAB provides the same results with a greater computation time.
} 
which is different in our case. The discount factor $\beta=0.9896$ that is consistent with an annual interest rate of $4 \%$. We let the utility function be defined as Cobb-Douglas, $u(c, 1-l)=\theta \ln c+(1-\theta) \ln (1-l)$ where $\theta=0.357$ which produces a steady state labor value of 0.31 . The production function is Cobb-Douglas with $F(k, l)=k^{\alpha} l^{1-\alpha}$, setting $\alpha=0.4$ to match the labor's share in national income. Capital's depreciation rate $\delta=0.0196$. We consider the TFP shock process $z_{t}=\rho z_{t-1}+\varepsilon_{t}$, where $\varepsilon_{t} \sim N\left(0, \sigma^{2}\right)$, and set $\rho=0.95$ and $\sigma=0.007$. We descritize the process into 9 states following Tauchen (1986) approximation. Our calibration procedure is summarized in Table 1 and 2 below.

\begin{tabular}{|c|c|c|c|c|c|c|c|c|c|}
\hline & \multicolumn{9}{|c|}{ Table 1: Calibration } \\
\hline & \multicolumn{7}{|c|}{ Discount factor } & \multicolumn{2}{|c|}{$\beta=0.9896$} \\
\hline & \multicolumn{7}{|c|}{ Consumption parameter in utility } & \multicolumn{2}{|c|}{$\theta=0.357$} \\
\hline & \multicolumn{7}{|c|}{ Share of capital in production } & \multicolumn{2}{|l|}{$\alpha=0.4$} \\
\hline & \multicolumn{7}{|c|}{ Depreciation rate } & \multicolumn{2}{|c|}{$\delta=0.0196$} \\
\hline & \multicolumn{7}{|c|}{ Persistence of the TFP shock process } & \multicolumn{2}{|l|}{$\rho=0.95$} \\
\hline & \multicolumn{7}{|c|}{ Standard deviation of the innovations to the TFP shock } & \multicolumn{2}{|c|}{$\sigma=0.007$} \\
\hline \multicolumn{10}{|c|}{ Table 2: Markov process } \\
\hline \multicolumn{10}{|c|}{ TFP shocks } \\
\hline \multicolumn{10}{|c|}{$z=[-0.0673,-0.0504,-0.0336,-0.0168,0,0.0168,0.0336,0.0504,0.0673]$} \\
\hline \multicolumn{10}{|c|}{ Transition matrix } \\
\hline \multirow{9}{*}{$\Pi=$} & $\lceil 0.7644$ & 0.2347 & 0.0009 & 0 & 0 & 0 & 0 & 0 & 0 \\
\hline & 0.0592 & 0.7405 & 0.1997 & 0.0006 & 0 & 0 & 0 & 0 & 0 \\
\hline & 0.0001 & 0.0747 & 0.7569 & 0.1679 & 0.0004 & 0 & 0 & 0 & 0 \\
\hline & 0 & 0.0001 & 0.0931 & 0.7669 & 0.1396 & 0.0002 & 0 & 0 & 0 \\
\hline & 0 & 0 & 0.0002 & 0.1147 & 0.7702 & 0.1147 & 0.0002 & 0 & 0 \\
\hline & 0 & 0 & 0 & 0.0002 & 0.1396 & 0.7669 & 0.0931 & 0.0001 & 0 \\
\hline & 0 & 0 & 0 & 0 & 0.0004 & 0.1679 & 0.7569 & 0.0747 & 0.0001 \\
\hline & 0 & 0 & 0 & 0 & 0 & 0.0006 & 0.1997 & 0.7405 & 0.0592 \\
\hline & 0 & 0 & 0 & 0 & 0 & 0 & 0.0009 & 0.2347 & 0.7644 \\
\hline
\end{tabular}

\subsubsection{Numerical results}

We follow the standard procedure in the literature (e.g. Judd (1992), Aruoba et al. (2006) and Barillas and Fernandez-Villaverde (2007)) to assess the accuracy of our solutions. To this end, we use the normalized Euler equation errors implied by the decision rules. In particular, with the current functional specifications, we compute the intertemporal Euler equation errors from (3) and (4), respectively

$$
\epsilon\left(k_{t}, z_{t}\right) \equiv 1-\frac{\theta\left(\beta \mathbb{E}_{z_{t+1} \mid z_{t}} u_{c}\left(g_{c}\left(g_{k}\left(k_{t}, z_{t}\right), z_{t+1}\right)\right)\left[e^{z_{t+1}} F_{k}\left(g_{k}\left(k_{t}, z_{t}\right), g_{l}\left(g_{k}\left(k_{t}, z_{t}\right), z_{t+1}\right)\right)+1-\delta\right]\right)^{-1}}{g_{c}\left(k_{t}, z_{t}\right)}
$$

which are expressed in units of consumption. Notice that the intratemporal Euler equation errors are always zero in both methods since labor is found with an exact solution, hence we do not report them. The 
Euler equation error provides a straightforward interpretation. For instance, an error of $10^{-3}$, or -3 units of the base-10 logarithm, means a mistake of $\$ 1$ for $\$ 1000$ spent. We calculate the maximum and mean Euler equation errors from the simulation of the model. ${ }^{10}$ We bring the MRM and PFI to the same standards by interpolating our decision rules from MRM over the grid points of capital used in PFI, so the decision rules are defined over the same grid points across the two different methods.

We use MATLAB software version 8.3.0.532 (R2014a) on a Lenovo Thinkpad with Intel(R) Core(TM) i7-4600U CPU $2.10 \mathrm{GHz}(2.69 \mathrm{GHz})$. Our codes are available for the reproduction of the results.

We use linearly-spaced grids and consider the performance of both methods under a range of different number of grid points with $k_{1}=0.3 k^{s s}$ and $k_{M}=1.9 k^{s s}$ where $k^{s s}$ is the steady-state level of capital. As shown in the results in Table 3, the MRM algorithm does not require a large number of grids. We obtain robust results across all grid sizes as MRM involves mostly exact solutions with nonlinear equation solvers and algebraic operations. When the number of nodes is 50, convergence is achieved in 193 iterations and 2.19 CPU seconds. For PFI, the results seem rather mixed. Among these, when the grid size is 1000 the mean Euler equation error is lower. In any case, MRM dominates PFI either in terms of speed or accuracy, or both. In Figures 1 and 2 in the Appendix, we also plot the policy functions from each algorithm, to provide further comparison.

\begin{tabular}{c|cccc|cccc}
\hline \hline \multicolumn{1}{c|}{ Table 3: Numerical results } \\
\hline \multirow{2}{*}{ Grids } & \multicolumn{7}{c|}{ MRM } & \multicolumn{4}{c}{ PFI } \\
\cline { 2 - 9 } & CPU & $\mathrm{L}_{1}$ & $\mathrm{~L}_{\infty}$ & Iterations & CPU & $\mathrm{L}_{1}$ & $\mathrm{~L}_{\infty}$ & Iterations \\
\hline 50 & 2.19 & -3.15 & -2.96 & 193 & 0.65 & -2.93 & -2.19 & 13 \\
100 & 4.26 & -3.15 & -2.96 & 193 & 2.63 & -2.99 & -0.82 & 16 \\
500 & 45.56 & -3.15 & -2.96 & 193 & 102.86 & -2.45 & -1.54 & 18 \\
1000 & 89.17 & -3.15 & -2.96 & 193 & 642.02 & -3.27 & -1.82 & 18 \\
\hline
\end{tabular}

Note: $L_{1}$ and $L_{\infty}$ are average and maximum of absolute Euler equation errors (in log 10 units), respectively. Errors are obtained from a stochastic simulation of 10,000 periods. CPU is the time necessary for computing a solution under a given method (in seconds).

The MRM algorithm requires more iterations for convergence. However, the total time spent for the solution of the problem shows that each iteration is completed faster compared to the time spent for each iteration in PFI. The speed in MRM can be attributed to (i) the time convention of grid points such that the grids are defined in terms of tomorrow's capital rather than today's capital (ii) the solution of capital and labor for the current period is made only once, and only at the end of the algorithm when convergence is achieved.

Finally, we compare the two approaches in terms of the complexity of implementation, as measured by the number of lines of the code. We only report the lines from the code solving the problem, since all the rest of the code is identical for both MRM and PFI. MRM requires 184 lines of code, 143 of which are for the subroutines that solve nonlinear equations (the csolve code of Christopher Sims and our Newton's method applied twice), hence the core algorithm is very easy to implement (41 lines). PFI requires a total of 94 lines of code, 32 lines of which is for Newton's method. Hence the main part of PFI requires 62 lines.

\footnotetext{
${ }^{10}$ We simulate the economy for 10,000 periods.
} 


\subsection{Conclusion}

The presence of an endogeneous labor-leisure choice might complicate the solution at the expense of computation time and/or accuracy. In this paper, we propose an easy-to-implement solution technique that circumvents the problems of nonlinear equation solvers by changing the time convention of the endogenous state variable, redefinition of the state variable as the end-of-period market resources and iterating over the policy function. We show numerical results based on the stochastic neoclassical growth model and accordingly, our proposed tecnhnique outperforms another powerful benchmark, Howard's policy improvement algorithm, in terms of speed, accuracy or both. 


\section{References}

Aiyagari, S. R. (1994). Uninsured Idiosyncratic Risk and Aggregate Saving. Quarterly Journal of Economics 109, 659-84.

Aruoba, S. B., J. Fernández-Villaverde, and J. F. Rubio-Ramírez (2006). Comparing Solution Methods for Dynamic Equilibrium Economies. Journal of Economic Dynamics and Control 30(12), 2477-2508.

Barillas, F. and J. Fernandez-Villaverde (2007). A Generalization of the Endogenous Grid Method. Journal of Economic Dynamics and Control 31, 2698-2712.

Bewley, T. F. (1986). Stationary Monetary Equilibrium with a Continuum of Independently Fluctuating Consumers. In W. Hildenbrand and A. Mas-Colell (Eds.), Contributions to Mathematical Economics in Honor of Gerard Debreu, pp. 79-102. North Holland.

Carroll, C. D. (2006). The Method of Endogenous Grid for Solving Dynamic Optimization Problems. Economics Letters (312-320).

Coleman, W. J. (1990). Solving the Stochastic Growth Model by Policy Function Iteration. Journal of Business and Economic Statistics 8(1), 27-29.

Cooley, T. and E. C. Prescott (1995). Economic Growth in Business Cycles. In T. F. Cooley (Ed.), Frontiers of Business Cycle Research, pp. 1-38. Princeton, NJ: Princeton University Press.

den Haan, W. J. and A. Marcet (1990). Solving the Stochastic Growth Model by Parameterizing Expectations. Journal of Business and Economic Statistics 8(1), 31-34.

Fella, G. (2014). A Generalized Endogenous Grid Method for Non-Smooth and Non-Concave Problems. Review of Economic Dynamics 17(2), 329-344.

Guerrieri, V. and G. Lorenzoni (2015, January). Credit Crisis, Precautionary Savings, and the Liquidity Trap. Mimeo.

Hintermaier, T. and W. Koeniger (2010). The Method of Endogenous Gridpoints with Occasionally Binding Constraints among Endogenous Variables. Journal of Economic Dynamics and Control 34, 2074-2088.

Howard, R. A. (1960). Dynamic Programming and Markov Processes. Cambridge, MA: MIT Press.

Huggett, M. (1993). The Risk-Free Rate in Heterogeneous-Agent Incomplete-Insurance Economies. Journal of Economic Dynamics and Control 17, 953-970.

Imrohoroglu, A. (1989). Cost of Business Cycles with Indivisibilities and Liquidity Constraints. Journal of Political Economy 97(6), 1364-83.

Judd, K. L. (1992). Projection Methods for Solving Aggregate Growth Models. Journal of Economic Theory (58), 410-452.

Judd, K. L. (1998). Numerical Methods in Economics. Cambridge, MA: MIT Press.

Kabukcuoglu, A. (2015). The Winners and Losers of Tax Reform: An Assessment under Financial Integration. Mimeo.

Kydland, F. and E. C. Prescott (1982). Time to Build and Aggregate Fluctuations. Econometrica 50(6), 1345-1370. 
Ljungquist, L. and T. Sargent (2012). Recursive Macroeconomic Theory. MIT Press.

Maliar, L. and S. Maliar (2013). Envelope Condition Method versus Endogenous Grid Method for Solving Dynamic Programming Problems. Economics Letters, 262-266.

Maliar, L. and S. Maliar (2014). Numerical Methods for Large Scale Dynamic Economic Models, Volume 3, Chapter 7, Handbook of Computational Economics, pp. 325-477. Amsterdam: Elsevier Science.

Puterman, M. L. and S. L. Brumelle (1979). On the Convergence of Policy Iteration in Stationary Dynamic Programming. Mathematics of Operations Research 4(1), 60-69.

Rendahl, P. (2015). Inequality Constraints and Euler Equation-Based Solution Methods. The Economic Journal 125, 1110-1135.

Santos, M. S. and J. Rust (2003). Convergence Properties of Policy Iteration. SIAM Journal on Control and Optimization 42(6), 2094-115.

Tauchen, G. (1986). Finite State Markov-Chain Approximations to Univariate and Vector Autoregressions. Economics Letters 20, 177-181.

White, M. N. (2015). The Method of Endogenous Gridpoints in Theory and Practice. Journal of Economic Dynamics and Control 60, 26-41. 


\section{Appendix}
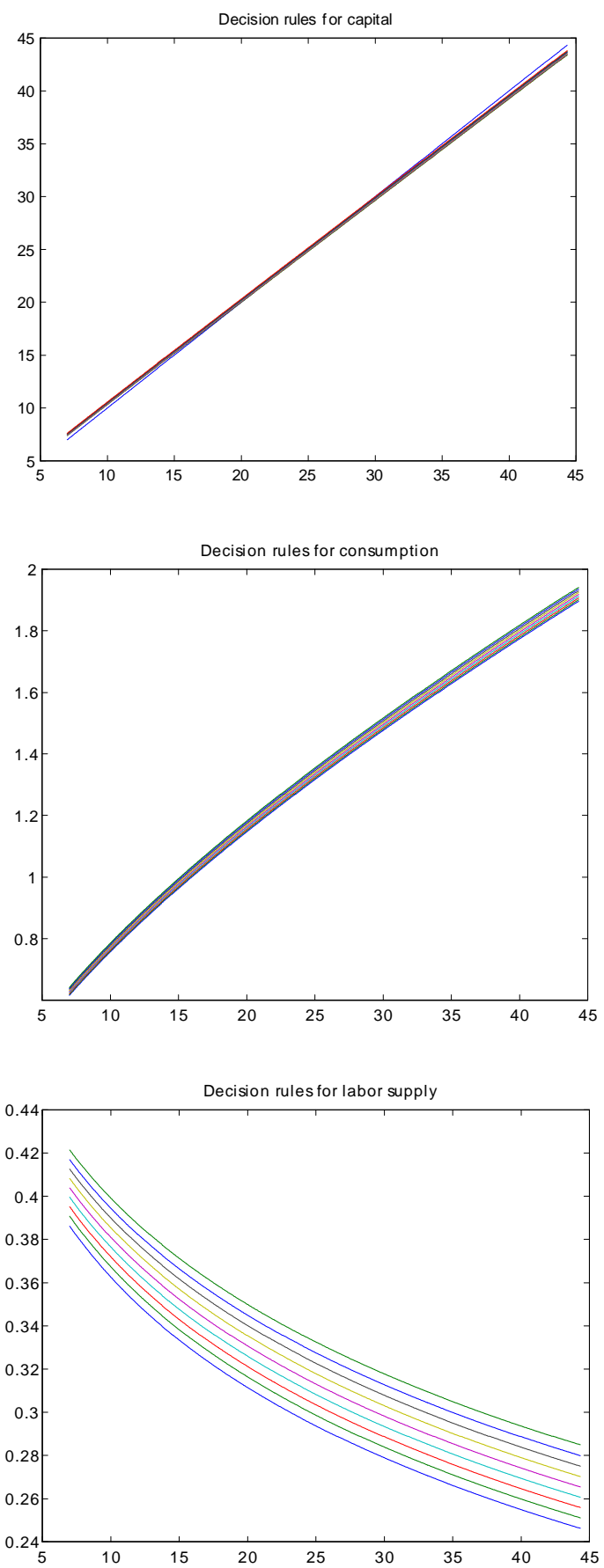

Figure 1: Decision rules obtained under MRM with 1000 capital grid points and 9 Tauchen nodes. 

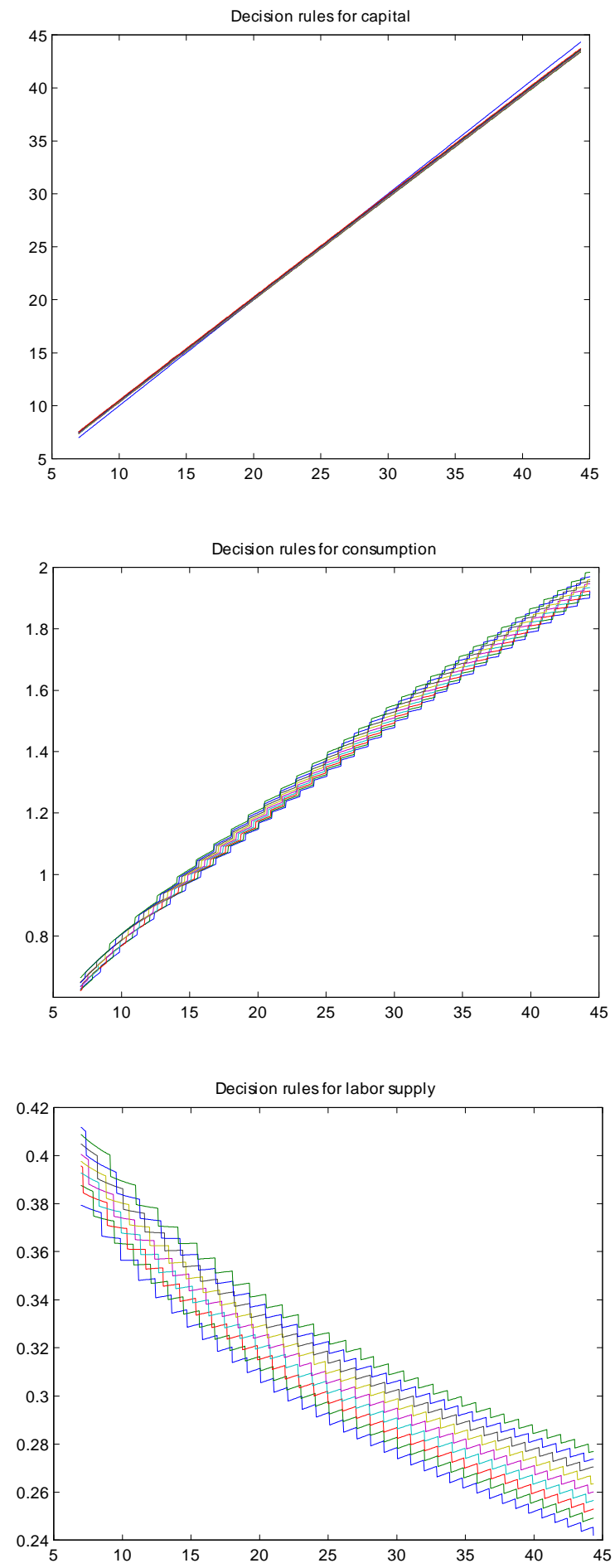

Figure 2: Decision rules obtained under PFI with 1000 capital grid points and 9 Tauchen nodes. 